\title{
L'énigme de la patate douce. Scénarios historiographiques dans le Pacifique
}

Jean-François Baré

\section{OpenEdition}

12 Journals

Édition électronique

URL : http://journals.openedition.org/jso/6438

DOI : $10.4000 /$ jso.6438

ISSN : $1760-7256$

Éditeur

Société des océanistes

\section{Édition imprimée}

Date de publication : 15 décembre 2011

Pagination : 415-422

ISBN : 978-2-85430-037-4

ISSN : 0300-953x

Référence électronique

Jean-François Baré, "L'énigme de la patate douce. Scénarios historiographiques dans le Pacifique », Journal de la Société des Océanistes [En ligne], 133 | 2e semestre 2011, mis en ligne le 31 décembre 2011, consulté le 23 septembre 2020. URL : http://journals.openedition.org/jso/6438 ; DOI : https:// doi.org/10.4000/jso.6438 


\section{L'énigme de la patate douce. Scénarios historiographiques dans le Pacifique}

par

Jean-François BARÉ*

La parution récente (2005) du livre édité par Chris Ballard, Paula Brown, R. Michael Bourke et Tracy Harwood, The Sweet potato in Oceania: a reappraisal ${ }^{1}$, m'amène à témoigner de ce que je crois être son grand intérêt. L' « énigme " de l'introduction de la patate douce dans le Pacifique Sud (et, éventuellement comme on le verra, en Asie du Sud-Est) depuis l'Amérique andine et/ ou le Mexique a longtemps fait l'objet de débats de rigueur variable (y compris dans ses prétendues résolutions les plus contestables, ainsi chez Thor Heyerdahl [1952]). On peut donc espérer qu'un résumé de l'exposé tout à fois pédagogique et rigoureux de Chris Ballard et de ses collaborateurs suscite le même genre d'intérêt.

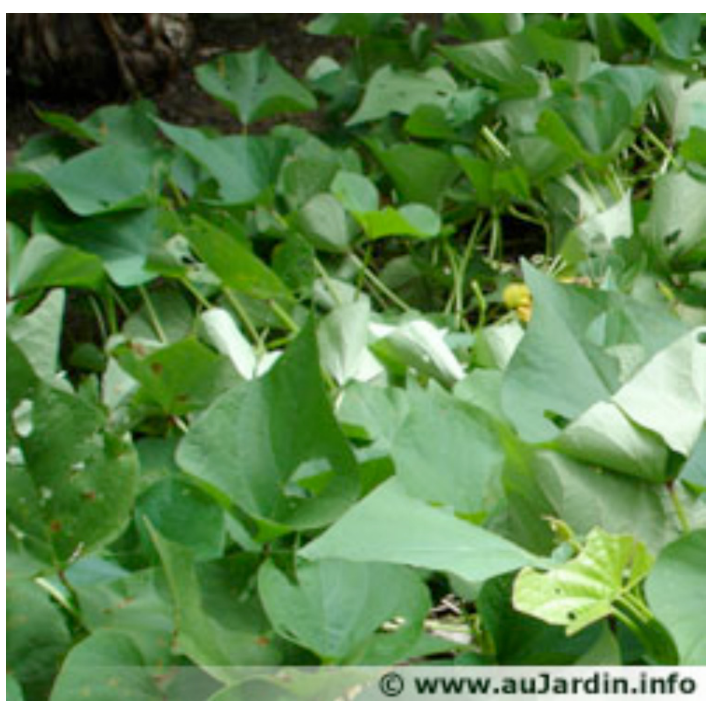

Rappelons que Ipomea batatas a depuis fort longtemps été considérée comme non indigène aux îles du Pacifique Sud, où elle a pourtant joué un rôle très important dans l'alimentation, depuis les premiers contacts européens ; ceci, notamment, du fait de l'homonymie entre le quechua kumar et le kumara (ou 'umara) de diverses langues notamment polynésiennes. Mais aussi, vu cette troublante indication, du fait que les plus anciennes traces d'une patate douce domestiquée viennent du Pérou, aux environs de 2000 avant J.-C. (O’Brien, 2000, cité par Montenegro et al., 2008 : 355). Un tubercule fossile de patate douce a même été trouvé dans le canyon de Chilca, toujours au Pérou, et daté de plus ou moins 8080 B.C. ; mais les archéologues ne sont pas sûrs qu'il s'agisse de patate douce domestiquée (Engel, 1970, cité par Montenegro et al., ibid.).

La question était alors : comment est-elle parvenue dans le Pacifique Sud ? Cette question a fait

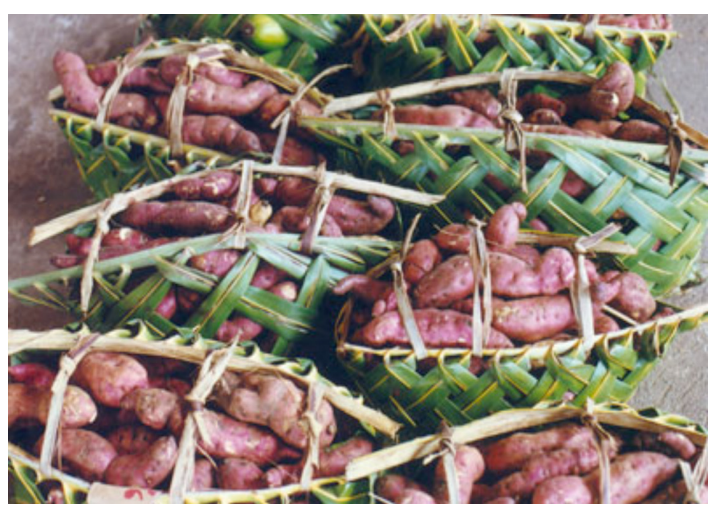

Рнотоs 1-2. - Le " corps du délit " : 1. Les feuilles (@) www.auJardin.info) ; 2. Les tubercules récoltés (CAnnie Walter, IRD, tous droits réservés)

1. Il sera noté ci-dessous Ballard et al., 2005.

* IRD et Université Paris I-Panthéon Sorbonne, uMr 201 Développement et sociétés, Jean-Francois.Baré@ird.fr. 
l'objet d'un nombre considérable de tentatives de réponses qui se sont elles-mêmes heurtées à autant de contre-arguments. Un relatif consensus s'est cependant longtemps établi sur l'origine sud-américaine de la plante (nord-ouest du Pérou puis, plus récemment, ouest du Mexique (Ballard et al., 2005 ; Montenegro et al., 2008). Ceci posé, pour résumer sommairement les principaux aspects du problème, les options étaient au nombre de quatre.

1. La patate douce a été introduite par les Espagnols depuis leurs possessions d'Amérique du Sud (c'est, dès 1786, la position de George Forster, frère cadet du naturaliste de Cook lors de son troisième voyage, dans sa thèse De Plantis Esculentis Insularum Oceani Australis, cf. Yen in Ballard et al., 2005), mais elle concerne dans ce cas les Philippines et les "Indes orientales". Pour Tahiti, l'un des points de "l'ellipse de diffusion " probable de Yen (1974), cette hypothèse ne tient pas car, ainsi que je l'ai vérifié, la plante y est attestée par Cook dès 1769 ainsi qu'en Nouvelle-Zélande maori la même année (Beaglehole [ed.], 1955 : 183 notamment); alors que les premières expéditions espagnoles, qui se sont accompagnées de nombreux échanges avec Lima y compris des visites de Tahitiens, datent de 1772 (Corney [ed.], 1913 ; voir aussi Salmond, 2009 : chap. 11 et 15).

2. La patate douce a été introduite suite à des contacts maritimes pré-européens entre la Polynésie et l'Amérique du Sud, soit qu'il s’agisse : 2.1. de contacts depuis l'Amérique du Sud.

C'est, on le sait la thèse, contestable et contestée d'Heyerdahl (1952), suivie de comparaisons incontrôlées entre, par exemple, les monuments inca et ceux de l'île de Pâques, comme on aimait les pratiquer à l'époque ; bien que Heyerdahl n’ait jamais véritablement soutenu le peuplement du Pacifique depuis l'Est, comme le note Ballard (1985a). Cela a donné lieu, dix étages intellectuels audessous, à de stupéfiantes élucubrations sur le peuplement « inca » du Pacifique (et même du Japon !) qui sévissent encore de nos jours, publiées par... Gallimard, dans la collection "l'Aube des Peuples » dirigée par... un prix Nobel de littérature, J.-M. G. Le Clézio (Baré, 2009) ;

2.2. de dérives accidentelles depuis les deux régions, mais plus probablement depuis l'Amérique du Sud du fait du régime des vents et des courants, à partir du même genre de radeaux en balsa que le Kon Tiki (nommé ainsi, rappelons-le, du fait de l'homonymie, peut-être de hasard, entre un dieu inca et les tiki polynésiens).
3. Une introduction par des diffusions subspontanées de graines, soit par la mer, soit par des oiseaux de mer, le problème étant au moins dans le premier cas les capacités plausibles de ces graines à survivre à un temps à reconstituer pour "la traversée ", ensuite leurs capacités à se reproduire sans intervention humaine (Montenegro et al., 2008, et ci-dessous). La patate douce est en effet généralement reproduite par " clonage " (prélèvement sur un tubercule déjà existant). Pour des raisons sur lesquelles on reviendra, le consensus semble se faire sur l'intervention humaine (buman agency), notamment in Ballard et al. (2005) (Ballard, 2005a : 3).

4. L'analyse ADN étant venue entretemps compliquer un peu plus le problème, les génomes des patates douces d'Océanie et d'Amérique du Sud sont considérés dans certains cas comme relativement distincts, dans d'autres comme relativement semblables, selon que les cultivars sont " préhistoriques " (comme en Nouvelle-Zélande) ou post-européens (Montenegro et al., 2008 : 357, citant Harvey et al., 1997).

Comme on le voit, le problème de l'introduction de la patate douce dans le Pacifique Sud constitue un extraordinaire " nœud " d' " intrigues » historiques au sens de Paul Veyne (1971), ce que j'appelle ici des scénarios. Pour reprendre l'exacte expression du titre de l'introduction de Chris Ballard, à qui ces lignes doivent beaucoup, la patate douce est toujours "bonne à penser " (still good to think with), selon l'expression célèbre de Claude Lévi-Strauss à propos des mythes. Le problème de son introduction dans le Pacifique montre que la question de la "vérité " (je crois bien qu'elle existe quelque part, contrairement aux relativistes de toutes obédiences!), est ellemême soumise au raisonnement historique, quelle que soit désormais la réelle scientificité des méthodes désormais employées (analyses ADN, modélisation des vents et des courants, etc.). Elle ressort donc finalement à des problèmes méthodologiques assez analogues à ceux de l'écriture de l'histoire au sens de Veyne.

J'aurais d'ailleurs tendance à penser que, de ce fait même et donc du fait des interprétations à l'œuvre, cette question puisse engendrer, y compris chez les natifs du Pacifique insulaire, des sortes de "champs politiques " (ou micro-politiques) au sens de Schwartz, Turner et Tuden (1968), qui n'ont parfois plus grand chose à voir avec le problème lui-même.

Ainsi, de la part, par exemple, de Tahitiens " ruraux ", on entend dire, ce qui est parfaitement compréhensible, que :

" bien sûr que la patate douce est fondamentalement indigène (mäobi), c'est quoi ces histoires ?" 
J'ai longtemps ignoré, par exemple, que la tomate était originaire elle aussi d'Ámérique. On peut trouver à l'inverse une sorte de halo mystérieux autour des liens avec l'Amérique du Sud, qui doit satisfaire les aspirations romantiques des uns ou des autres et qui résiste passionnément au doute.

Pour faire part à titre d'illustration de mon expérience personnelle, j'eus ainsi l'occasion à Tahiti, dans les années 1975-1980, de dîner en compagnie de ce que l'on appelait à l'époque un " conseiller de gouvernement " chargé de la Culture (que l'on appelle désormais un " ministre »). Alors que la conversation s'en vint tourner autour de l'expédition du Kon Tiki (souvent considérée localement comme l'un de ces exploits légendaires propres à la navigation sur grande distance), j'eus la juvénile imprudence de faire part de mes doutes sur la seule occurrence commune de kumara à la fois en Amérique latine et dans le Pacifique. "Je ne crois pas", dis-je en gros et trop professoralement " qu'un seul élément puisse faire beaucoup de sens pour un peuplement depuis l'Amérique latine». Ce propos jeta un froid et mon hôtesse et amie me réprimanda un peu en partant : " tu sais, avec tes histoires de kumara, je t'ai vu mal parti ». J'appris par la suite que le conseiller, qui se disait « autonomiste " selon le vocabulaire de l'époque, était fort proche de Bengt Daniellsson, " autonomiste " également, compagnon de Thor Heyerdahl sur le Kon Tiki, farouche ennemi (pour des raisons que je n'ai pas à discuter) des essais nucléaires et auteur avec son épouse Marie-Thérèse du livre connu Moruroa mon amour. Tout ceci n'a apparemment pas grand chose à voir avec l'origine de la patate douce; mais cette énigme attire irrésistiblement ce genre de liaisons logiques.

De même, quand je lui adressai récemment des critiques très directes sur un article évoquant, de manière à vrai dire parfaitement creuse, le peuplement de la Polynésie depuis l'Amérique du Sud, le directeur d'un hebdomadaire publié à Tahiti m’objecta avec quelque dérision " la célébration mondiale de Thor Heyerdahl "; étant mondialement célèbre, il devait donc avoir raison.

À présent, quelles sont les réponses possibles, compte tenu des dernières avancées en ces domaines (archéologie et analyses ADN notamment) au corps d'hypothèses évoquées ?

Pour Ballard (2005a), le travail de Douglas Yen (1974), qui signe d'ailleurs le chapitre final du volume commenté ici, constitue un jalon décisif pour fixer le cadre des hypothèses historiques. Yen note que l'origine sud-américaine de la patate douce avait été évoquée dès 1886 par le botaniste suisse Alphonse de Candolle. Un consensus sur ce point semblait néanmoins s'être fixé dès avant le travail de Yen, qui l'a confirmé. Les régions concernées sont le Nord-Ouest de l'Amérique latine (Nord du Pérou) et la côte Ouest du Mexique (plus probablement SudOuest) (notamment Scaglion, in Ballard et al., 2005 : chap. IV).

Le consensus était beaucoup moins net sur les modalités de diffusion : sub-spontanées ou humaines ? Sur ce point, le travail de Yen a nettement tranché en faveur de la diffusion humaine, dont l'hypothèse semble à nouveau confirmée notamment par Roger Green dans ce volume. Il en va ainsi dans un non moins intéressant article d'Helen Leach, autrefois adepte d'une diffusion sub-spontanée. Elle note qu'en l'absence d'une connaissance des modes de culture de la plante lors de son introduction, ce qui suscitait l'essentiel de ses doutes, elle a probablement dû être traitée comme une sorte d'igname métaphorique (ufi dans beaucoup de langues polynésiennes).

Ballard note l'existence d'un "âge d'or " du débat sur l'hypothèse de la diffusion humaine, datant comme on l'a vu au moins de l'époque de Cook, mais particulièrement intense à la première moitié du $\mathrm{XX}^{\mathrm{e}}$ siècle. Il avait généralement pour point commun de discuter d'options alternatives, les Espagnols, les Polynésiens ou les Amérindiens euxmềmes. Le débat fut bien sûr relancé par la spectaculaire expédition de Thor Heyerdahl.

L'ethno-botaniste Jacques Barrau (1957) fut cependant le premier à concilier les extrêmes, si l'on ose dire, en proposant une hypothèse de diffusion tripartite :

- l'itinéraire kumara (kumara line) (de l'Amérique du Sud en Polynésie, par les Amérindiens ou les Polynésiens),

- l'itinéraire kamote (kamote line, depuis le Mexique vers l'Asie du Sud-Est par les Espagnols, du philippin kamote)

- et l'itinéraire batata (d'un autre nom quechua de la patate douce) ou batata line liée au transfert par les Portuguais vers l'Europe (et les Caraïbes et l'Asie), celui-là même qui donna naissance à la fameuse "patate " de Parmentier. Il intégrait ainsi une première hypothèse bipartite due à Hornell (1946) qui omettait l'itinéraire batata ${ }^{3}$.

Ainsi naquit une carte de diffusion de la patate douce, que Ballard nous dit désormais célèbre et qui figure déjà dans ses grandes lignes dans Yen 1974. Pour discuter de la possibilité des itinéraires plausibles, il fallait en effet les connaitre.

Cette carte célèbre fut ensuite raffinée par le même auteur en 1982 comme suit :

S'agissant de "l'itinéraire kumara", un consensus assez large, mais peu argumenté à l'époque,

2. Le mot kamote provient en fait du nahuatl ou du mixtèque (voir Hildebrandt, 1969 : 72-73, com. pers. du géographe Jean-Paul Deler).

3. Notons que, dans le Nord-Ouest de Madagascar, la patate douce est appelée batata mamy (batata « sucrée "). 
sur la transmission dans le Pacifique Sud par les Polynésiens eux-mêmes, fut mis en cause, on l'a vu, par l'expédition du Kon Tiki. Yen reconnaissait certes que l'idée de voyages aller et retour depuis la Polynésie jusqu'en Amérique du Sud était plus compliquée à soutenir que l'introduction directe, mais son contre-argument était l'absence notable en Polynésie d'éléments essentiels de l'alimentation amérindienne, bien plus susceptibles que la patate douce d'être choisis pour des voyages à longue distance, comme le maïs ou les haricots du genre Phaseolus, entre autres cultures à semences (Yen, 1974 : 264267). Dans le volume édité par Ballard et al., les principales thématiques abordées par Yen sont à nouveau discutées : archéologiques, palynologiques, ou en linguistique diachronique, mythologie comparative, historiographie ar- chivistique. On aura noté que, sur la carte de Yen, un "sous-itinéraire " est indiqué en pointillés, vers la Nouvelle-Guinée. L'origine de la kumara line depuis cette île, parfois évoquée, a été largement mise en cause.

Le rôle de la patate douce en Nouvelle-Guinée est largement évoqué dans ce livre, dans le cadre d'analyses dites " sociologiques " par Ballard (adaptations, famines, réponse aux événements climatiques comme El Niño), dans neuf chapitres ${ }^{4}$, soit une bonne moitié du volume. Étant encore moins compétent sur la Nouvelle-Guinée qu'en ethnobotanique, je me contenterai de signaler ces pages à nos collègues spécialistes. Ballard écrit que sur l'" introduction " de la patate douce dans cette île, on est beaucoup plus qu'ailleurs dans un domaine " ressortant de la discussion purement conjecturale »(2005a : 7).

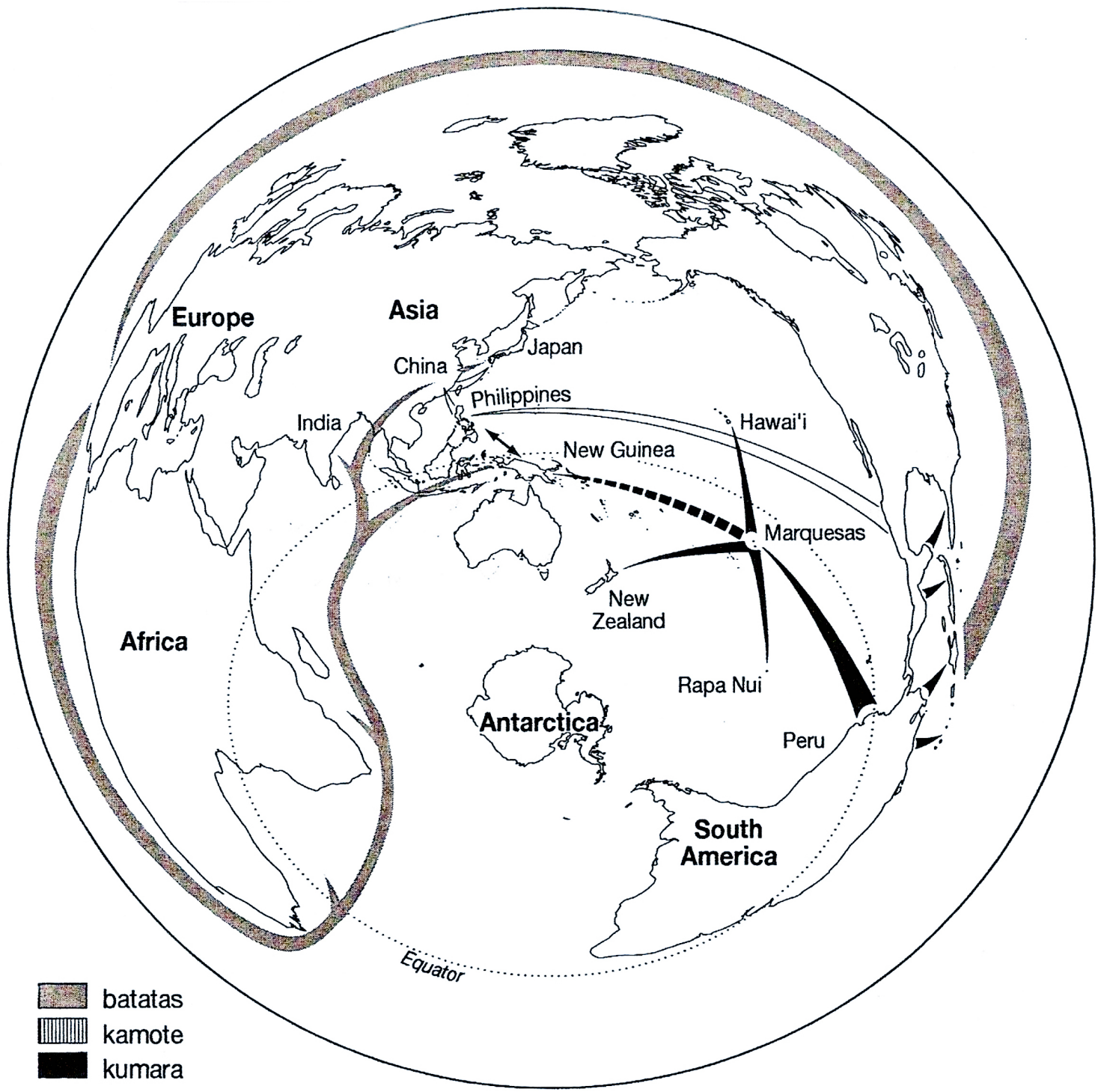

CARTE 1. - Itinéraires de diffusion de la carte de Yen (1982), in Ballard et al. (2005: 4) (tous droits réservés)

4. Voir les chapitres 10 : Allen ; 11 : Bayliss-Smith et al.; 12 : Wiesnner ; 13 : Brown et Brookfield ; 14 : Boyd ; 15 : Ploeg ; 16 : Yaku et Widyastuti ; 2 et 17 : Bourke, sur la « révolution ipoméenne ». 
Cependant, note Ballard, Yen « aurait probablement été déçu " par la relative incertitude des travaux archéologiques capables de valider de manière incontestable sinon ses "itinéraires" probables, du moins la nature et la chronologie de leurs directions. Certes, une datation incontestable était disponible dès 1974, pour le site de Lapakahi à Hawaii (entre 1358 et 1626 de notre ère (Rosendahl and Yen, 1971). Plus décisives encore, les fouilles de Hather et Kirch (1991) ont permis de dater le site de Mangaia, aux îles Cook, vers l'an 1000. Cette datation, bien antérieure à la découverte du Pacifique Sud au regard européen, élimine en effet l'idée d'une diffusion initiale par les Espagnols, mais pour partie seulement, car l'on ne peut évidemment exclure d'après moi son existence comme coextensive, par la suite, de « re- » diffusions de nouvelles souches, notamment par les Espagnols. (J'aurais tendance à penser que c'est effectivement le cas dans le Tahiti de 1772 ; voir notamment le chapitre de Salmond [2009] : « des Tahitiens à Lima ».)

Roger Green (ce volume), considéré sur ces questions comme un spécialiste éminent, semble en effet recueillir l'assentiment général sur une thèse longuement argumentée, celle de voyages polynésiens aller et retour vers l'Amérique du Sud, bien que Ballard note que " la nature du transfert initial depuis les Amériques reste problématique » (2005a: 6). Pour Green, l'hypothèse la plus vraisemblable est celle de voyages maritimes provenant d'une région située approximativement, soit vers Mangareva (les actuelles " Gambier »), soit à Rapa nui («l'île de Pâques»).

De fait, la possibilité de voyages vers l'Est c'est à dire généralement contre les vents dominants, sauf pour un bon tiers de l'année (les fameuses «brises d'ouest ", les westerlies de Cook) - est parfaitement attestée par ce que l'on sait désormais être le long peuplement d'ouest en est du Pacifique Sud aussi bien que par ce que l'on peut appeler les admirables " travaux pratiques " de Ben Finney (voir, par exemple, Ben Finney, 1985 ; voir aussi le site passionnant de la Polynesian Voyaging Society, http://pvs.hawaii.edu, dont il fut l'un des fondateurs).

Si l'on explore vers l'est l'immense Pacifique, disons depuis Mangareva (à 50 milles marins de progression par jour, hypothèse basse, soit quelque chose comme deux mois en progression directe pour environ 3000 milles), il est pratiquement impossible de "manquer" l'Amérique du Sud. Maintenant, comment a-t-on sélectionné puis rapporté la plante? L'hypothèse d'Helen Leach (ce volume) selon laquelle n'importe quel tubercule et notamment l'igname pouvaient " faire penser " à la patate douce me paraît toujours intéressante. Maintenant, les conditions de rencontre entre des proto-Polynésiens et des proto-Quechua restent pour l'instant mystérieuses :

\section{«Dr Livingstone, I presume?».}

Sur cette question, la solution du "retour" reste beaucoup plus aisée. Un article postérieur au livre de Ballard et al., article que je trouve personnellement remarquable (Montenegro et al., 2008) a réouvert deux discussions, celle de la diffusion sub-spontanée et celle de l'arrivée d'embarcations en Polynésie depuis l'Amérique du Sud, tout en acceptant globalement l'état actuel du savoir tel que résumé ci-dessus, notamment in Ballard et al. (2005). Il " modélise " en effet à partir de simulations sur ordinateur tenant compte de toutes les conditions climatiques possibles les probabilités d'une diffusion subspontanée et d'une diffusion humaine, par embarcations, mais en y opposant d'abord quelques arguments. Dans le premier cas, cette diffusion sub-spontanée n'aurait pas pu être due à des oiseaux de mer, comme le pluvier doré (Golden plover, Pluvialis appicaria) connu pour fréquenter les côtes de l'Amérique du Sud, mais inconnu en Polynésieoù l'on ne connaît que deux espèces de pluvier dans le Tahiti du XviII ${ }^{\mathrm{e}}$ siècle comme des manifestations ( $a t a$ ) des "dieux ", le pluvier "gris " (u'riti) évoquant les dieux de l'eau, le pluvier " siffleur" (torea) représentant Temeharo, " dieu " de la strangulation (voir Teuira Henry, 1928 : 364sq., citée et traduite depuis Oliver, 1974 : 59-60) ; mais de pluvier doré, apparemment point.

Bulmer (1966), cité par Montenegro et al. (2008 : 356), pensait à cette époque que le transport dans l'estomac des oiseaux de mer pouvait aider à la germination de graines dont ne sont généralement pas tirées directement, comme on l'a $\mathrm{vu}$, les patates douces. Mais il considérait comme improbable l'introduction par les oiseaux de mer, vu les distances en cause. Par contre, peut-on éliminer totalement l'hypothèse de dérives sur des débris de bois, ou encore de dérives accidentelles d'embarcations comme le Kon Tiki ? Montenenegro et al. citent différents témoignages anciens attestant des capacités de voyages à longue distance des radeaux en balsa de l'Équateur et du Nord du Pérou, de Lima jusqu'au golfe de $\mathrm{Pa}$ nama (par exemple, Edwards, 1965).

Un court croquis valant parfois mieux qu'un long discours, voici ce à quoi aboutit la modélisation de Montenegro et al., dans l'éventualité de la diffusion sub-spontanée ou dans celle de la diffusion humaine.

Bien que les auteurs notent que les probabilités d'occurrence sont difficiles à interpréter $(5$, $68 \%$ par exemple dans le cas des Marquises), cette simulation éveille irrésistiblement en moi une remarque sur ce que les océanographes appellent le " gyre " du Pacifique Sud, c'est-à-dire la conjonction, à partir du sud de l'Amérique du Sud (et non pas du Pérou il est vrai mais on s'y rejoint ensuite), des courants et des vents dominants vers le nord-ouest puis l'ouest. C'est ainsi 
que les premiers bateaux européens, qui remontaient très mal au vent (comme le Dolphin « découvreur " de Tahiti au regard européen [1767] ou bien, quelques décennies antérieurement, le Africaneschen Galley du Hollandais Jacob Roggeeven [1722]) se sont laissés dépaler par le courant de Humboldt depuis le cap Horn (ils ne pouvaient guère faire autrement), pour « attraper " la zone des alizés d'est et aboutir de ce fait, qui à Tahiti, qui aux Tuamotu (des centaines de kilomètres au nord) où Rogeeveen a d'ailleurs fait naufrage, comme c'était souvent le cas dans cette zone "mal pavée " pour les bateaux.

Pour résumer grossièrement mon impression à partir de raisonnements passionnants et sophistiqués (leurs auteurs me pardonneront peut-être), si l'on met n'importe quoi pouvant survivre disons deux ou trois mois à partir du milieu de la côte ouest de l'Amérique latine, cet objet parvient à Tahiti, aux Tuamotu et éventuellement

\section{Á. Montenegro et al. / Journal of Archaeological Science xx (2007) 1-13}

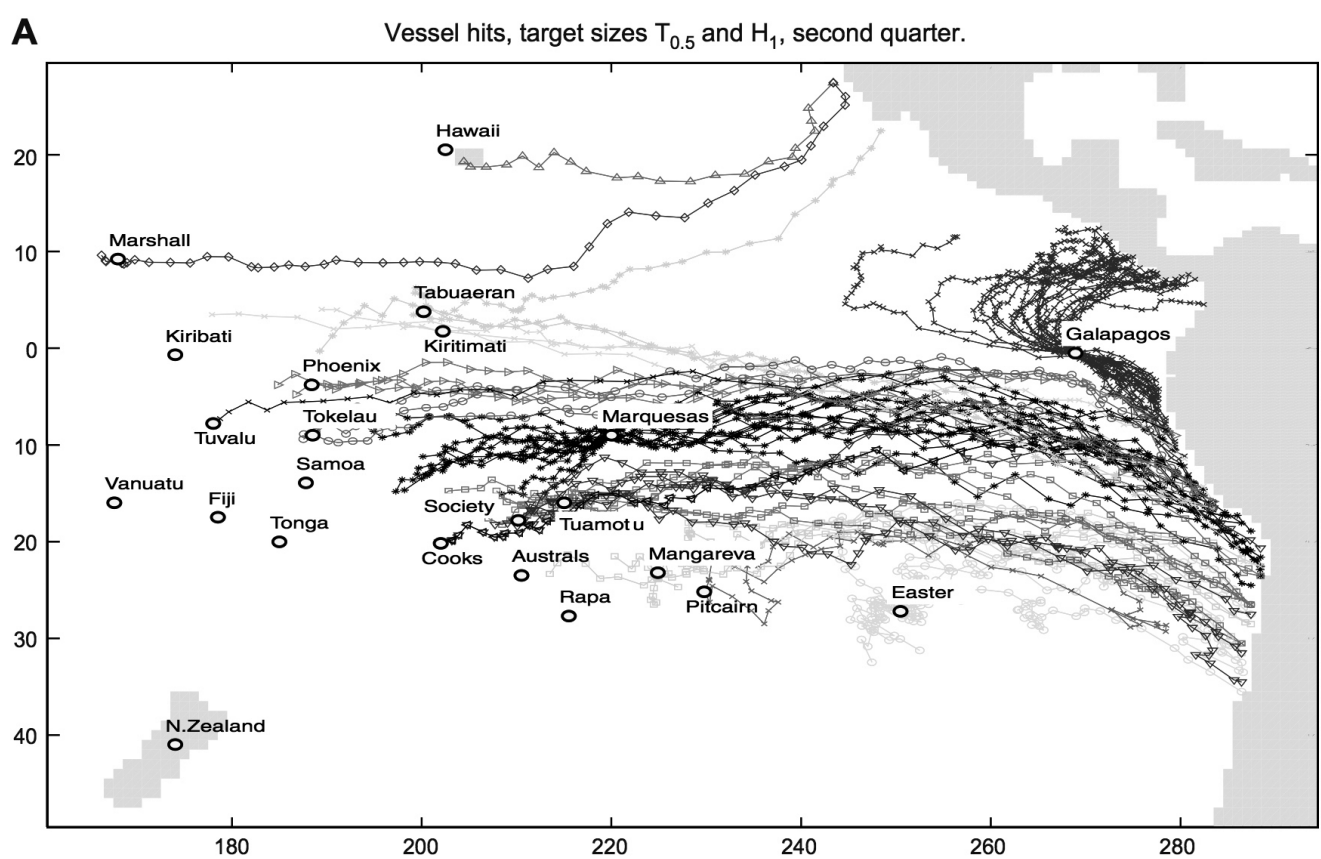

B Seed hits, target sizes $\mathrm{T}_{0.5}$ and $\mathrm{H}_{1}$, second quarter.

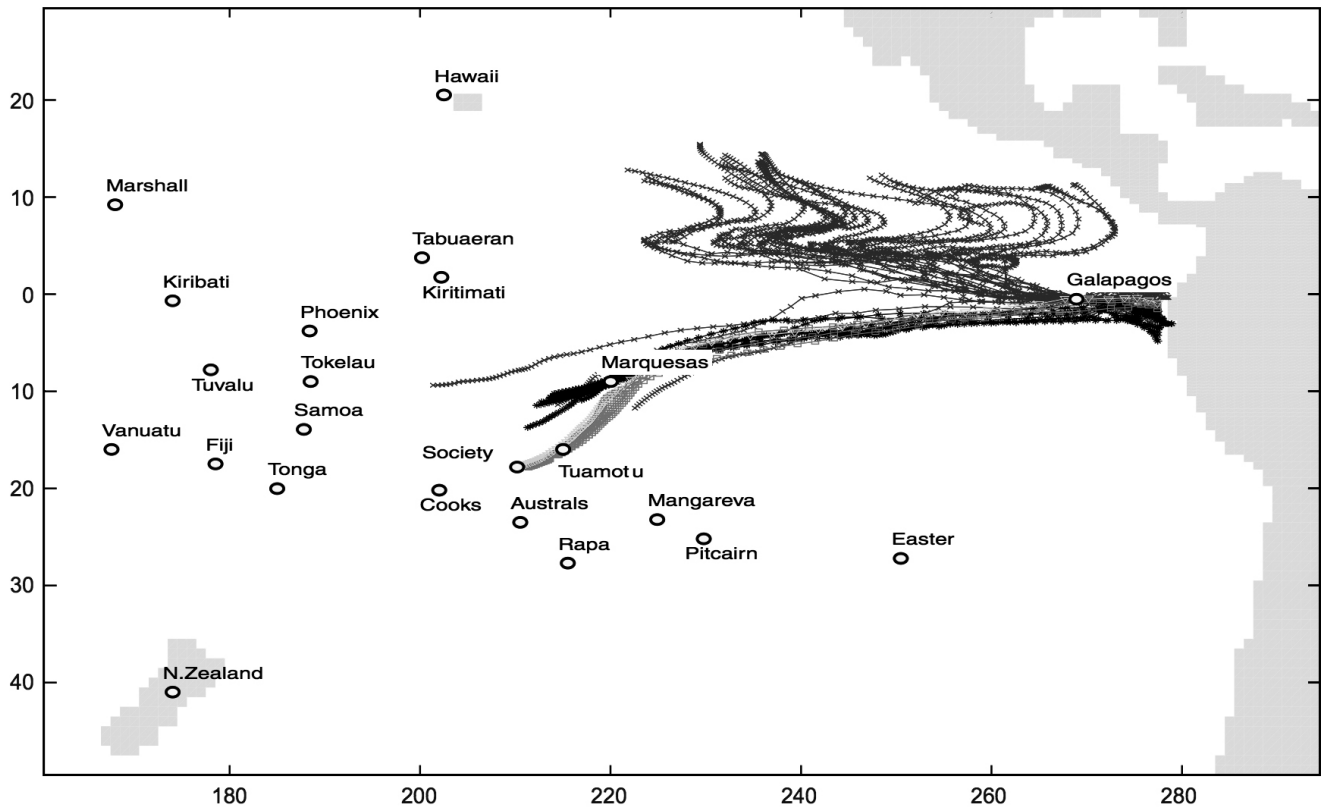

Figure 1. - Example of simulation results. Top: Track of the vessels that hit targets of size T.0.5 (for Hawaii,

Th1) in the second quarter experiment. Colors refer to the island where the hit occurred. Bottom: As for top panel for the seed capsule drift simulation (CMontenegro et al., $2008: 362$ ) 
aux Marquises. Les Galapagos, de manière plus surprenante mais pressentie je crois par Heyerdahl, sont également d'après Montenegro et al. (2008) sur un trajet prévisible.

Maintenant, qu'est ce que cet objet : des capsules de semence de patate douce ou bien Ipomea batatas dans toute sa majesté ? Il reste encore, si j'ose dire, "du pain sur la planche » mais je voudrais remercier Ballard et tous les auteurs spécialistes de nous avoir fait partager leur savoir. Comme le dit à peu près Paul Veyne (1971), en histoire (puisque que c'est finalement d'histoire qu'il s'agit, ou disons d'historiographie), il ne faut pas se poser la question de causalités linéaires, mais se dire " que vont-ils encore inventer ce coup-ci ? ». L'énigme de la patate douce m'en paraît une excellente illustration.

Bordeaux, mai-juin 2010

\section{Dédicace}

Quant à moi, j'aimerais dédicacer aussi ce texte à la mémoire de ma tendre épouse Annie $\mathrm{Hu}$ bert-Baré, ethnobotaniste dans l'âme s'il en fut, partie au jardin d'Eden sans avoir eu le temps de le lire ni de le critiquer.

\section{BIBLIOGRAPHIE}

BALlARD Chris, 2005a. Still good to think with: the sweet potato in Oceania, in Ballard et al., The sweet potato in Oceania: a reappraisal, Sydney, University of Pittsburgh - University of Sydney, Ethnology Monographs 19, Oceania Monographs 56, pp. 1-13.

Ballard Chris, Paula Brown, R. Michael Bourke and Tracy Harwood, 2005. The sweet potato in Oceania: a reappraisal, Sydney, University of Pittsburgh - University of Sydney, Ethnology Monographs 19, Oceania Monographs 56.

BARrau Jacques, 1957. L'énigme de la patate douce en Océanie, Études d'Outre-Mer 40, 3/4,

Baré Jean-François, 2009. Compte-rendu de Michel Brun, date, Eteroa Mythes, légendes et traditions d'une île polynésienne (Paris, Gallimard, «l'Aube des Peuples »), L'Homme 189, pp. 262-264

Beaglehole John Cawte (ed.), 1955. The Journals of Captain James Cook on His Voyages of Discovery, vol. 1, Cambridge, Cambridge University Press, Hakluyt Society Extra Series 34.
Bulmer Raph, 1966. Birds as possible agents in the propagation of the sweet potato, The Emu 65, pp. 165-182 (cité par Montenegro et al., 2008).

Candolle Adolphe (de), 1886. Origin of Cultivated plants, New-York, Hafner (réédition en 1959 de la $2^{\mathrm{e}}$ édition, cite par Yen, $1974: 1$ ).

Corney B. G. (ed.), 1913. The Quest and Occupation of Tahiti by Emissaries of Spain during the Years 1772-1776, told in Despatches and other Contemporary Documents I, II, and III, Cambridge for the Hakluyt Society.

Edwards C. R., 1965. Aborigenal Watercraft on the Pacific Coast of South America, Berkeley, University of California Press.

ENGEL Frédéric, 1970. Exploration of the Chilca Canyon, Peru, Current Anthropology 11, pp. 55-58.

FinNey Ben R., 1985. Anomalous westerlies. El Niño and the colonization of Polynesia, American Anthropologist 87, pp.9-26.

Green Roger, 2005. Sweet potato transfers in Oceania, in C. Ballard et al., The sweet potato in Oceania: a reappraisal, Sydney, University of Pittsburgh - University of Sydney, Ethnology Monographs 19, Oceania Monographs 56, pp. 43-62

Harvey Catherine F. G., Geffrey P. Gill, Catherine Crossman and Lean G. Fraser, 1997. Assessing relationships of kumara cultivars by RAPD Analysis, New Zealand Journal of Botany 35, pp.479-485.

Hather John G. and Patrick V. Kirch, 1991. Prehistoric sweet potato (Ipomea batatas) from Mangaia Island, Central Polynesia, $A n-$ tiquity 65, pp. 887-893.

Henry Teuira, 1928. Ancient Tahiti. Based on Material Recorded by John Muggridge Orsmond, Honolulu, Bernice P. Bishop Museum Bulletin 48.

Hornell J., 1946. How did the sweet potato reach Oceania?, Journal of the Linnean Society of London, Botany 53 (348), pp. 41-62.

Heyerdahl Thor 1952. American indians in the Pacific: The Theory Behind the Kon-Tiki Expedition, London, Allen and Unwin.

Hildebrandt Martha, 1969. Peruanismos, Lima, Moncloa,Campodonico.

LeACH Helen 2005. Ufi kumara, the sweet potato as yam, in C. Ballard et al., The sweet potato in Oceania: a reappraisal, Sydney, University of Pittsburgh - University of Sydney, 
Ethnology Monographs 19, Oceania Monographs 56, pp. 63-70.

Montenegro Alvaro Chris Avis and Andrew Weaver, 2008. Modeling the prehistoric arrival of the sweet potato in Polynesia, Journal of Archeological Science 35, pp. 355-367.

O'Brien Patricia, 2000. Sweet potatoes and Yams, in K.F. Kipple and K.C. Ornelas (eds.), The Cambridge World History, NewYork, Cambridge University Press, pp. IIB5.

Oliver Douglas, 1974. Ancient Tahitian Society, 3 vols, Honolulu, University of Hawaii Press.

Rosendahl Paul H. and Douglas E. Yen, 1971. Fossil sweet potatoes remains from Hawaii, Journal of the Polynesian Society 80 (3), pp. 379-385.

SALmond Ann, 2009. Aphrodite's Island. The European Discovery of Tahiti, Penguin, Viking.

SCAglion Richard, 2005. Kumara in the Ecuadorian Gulf of Guyaquil, in C. Ballard et al., The sweet potato in Oceania: a reappraisal, Syd- ney, University of Pittsburgh - University of Sydney, Ethnology Monographs 19, Oceania Monographs 56, pp. 35-41.

Schwartz Mark J., Victor W. Turner and Arthur Tuden, 1968. Political Anthropology, Chicago, Aldine Publishing Company.

Veyne Paul, 1971. Comment on écrit l'histoire, Paris, Seuil, " Points".

Yen Douglas E., 1974. The Sweet Potato and Oceania: An Essay in Ethnobotany, Honolulu, Bernice P. Museum Bulletin 236.

- 1982. Sweet potato in historical perspective, in R.L. Villareal and T.D. Griggs (eds), Sweet potato, Proceedings of the First International Symposium Asian Vegetable Research and Development Center, Taiwan, (non paginé)

-, 2005. Reflection, refraction and combination, in C. Ballard et al., The sweet potato in Oceania: a reappraisal, Sydney, University of Pittsburgh - University of Sydney, Ethnology Monographs 19, Oceania Monographs 56, pp. 181-188 